\title{
A Comparative Analysis of WSN Sensor Positioning Method using Iterative Routing Algorithm with Conventional Methods
}

\author{
N.Pushpalatha \\ Assistant Professor, Department of ECE \\ AITS, Tirupathi, India
}

\author{
B.Anuradha, Ph.D \\ Associate Professor, Department of ECE \\ S.V. University College of Engineering, Tirupathi ,India
}

\begin{abstract}
Wireless Sensor Networks (WSN) has been proposed as a solution to environment sensing, target tracking, data collection and other applications. Sensor positioning is one of the important problems in WSN. Strong antenna arrays at each node or sensor arrays at different positions are used to localize the sources. This paper proposes a sensor positioning algorithm for WSN. A comparative analysis is made for proposed method along with existing methods with respect to computational cost, shortest path, and number of nodes, radio range and ranging error. The results show that proposed method: iterative routing algorithm provides improved performance over existing methods in the aspects considered.
\end{abstract}

\section{Keywords}

Sensor Positioning, Computational Cost, Shortest Path, WSNs (Wireless Sensor Networks), Radio Range.

\section{INTRODUCTION}

Sensor nodes have the ability to sense the environment nearby, perform simple computations and communicate in a small region. Although their capacities are limited, combining these small sensors in large numbers provide a new technological platform, called Wireless Sensor Networks (WSNs) .WSNs provide reliable operations in various application areas including environmental monitoring, health monitoring, vehicle tracking system, military surveillance and earthquake observation [3-4].Although WSNs are used in many applications, they have several restrictions including limited energy supply and limited computation and communication abilities. In sensor networks, energy is a critical resource, while applications exhibit a limited set of characteristics. Thus, there is both a need and an opportunity to optimize the network architecture for the applications in order to minimize resource consumed. The requirements and limitations of sensor networks make their architecture and protocols both challenging and divergent from the needs of traditional Internet architecture. A sensor network [1] is a network of many tiny disposable low power devices called nodes, which are spatially distributed in order to perform an application-oriented global task. These nodes form a network by communicating with each other either directly or through other nodes. One or more nodes among them will serve as sink which are capable of communicating with the user either directly or through the existing wired networks. The primary component of the network is sensor, essential for monitoring real world physical conditions such as sound, temperature, humidity, intensity, vibration, pressure, motion, pollutants etc...at different locations. The tiny sensor nodes which consist of sensing on board processor for data processing, and communicating components, leverage the idea of sensor networks based on collaborative effort of a large number of nodes [5-6]. Figure 1 shows the structural view of a sensor network in which sensor nodes are shown as small circles. Each node typically consists of the four components: sensor unit, central processing unit (CPU), power unit, and communication unit. They are assigned with different tasks [7].

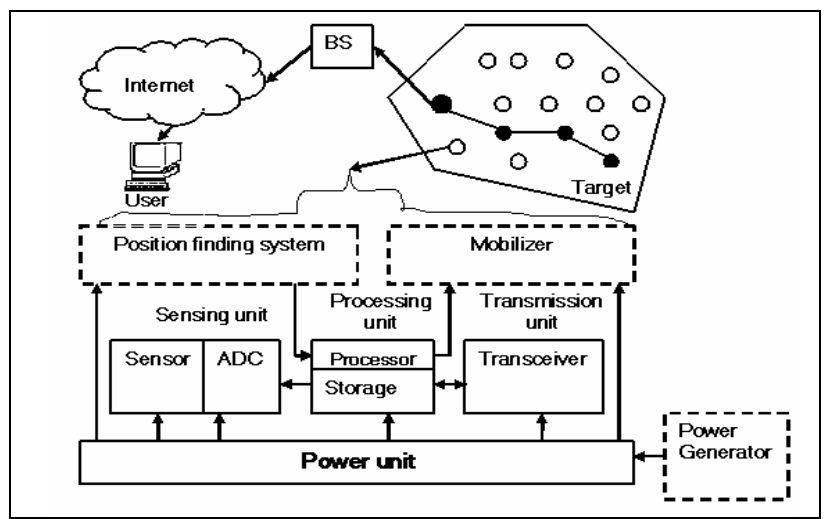

Fig 1: Structural view of a sensor network

The rest of this paper is organized as follows: Section II describes the brief idea about sensor positioning in WSNs. Section III describes a Comparison of IRA along with the conventional methods about ACO and PSO techniques. Section IV gives Simulation Results of the paper has been discussed. In section V Conclusion and Future work, where the future challenges and directions to improve localization in WSN technology are described.

\section{SENSOR POSITIONING IN WSN}

An IR Algorithm is a technique widely used for the analysis of dissimilarity of data on a set of objects, can discover the spatial structures in the data. It is used it as a data-analytic approach to discover the dimensions that underlie the judgments of distance and model data in a geometric space. The main advantage of using an IRA for position estimation is that it can always generate relatively high accurate position estimation even based on limited and error-prone distance information. It is also one of the methods in MDS algorithms. There are several varieties of routing algorithms to find out the shortest path between the source node and destination node for position estimation. The basic idea of this algorithm is to assume that the dissimilarity of data are distances and then deduce their coordinates. More details about comprehensive and intuitive explanation of MDS are available in [3]. Inspired by the above multidimensional scaling techniques, present a multivariate optimization based iterative algorithm for sensor location calculation is presented in this 
paper [2]. The flow chart shows the best rout from source to destination nodes as shown in Fig.2.

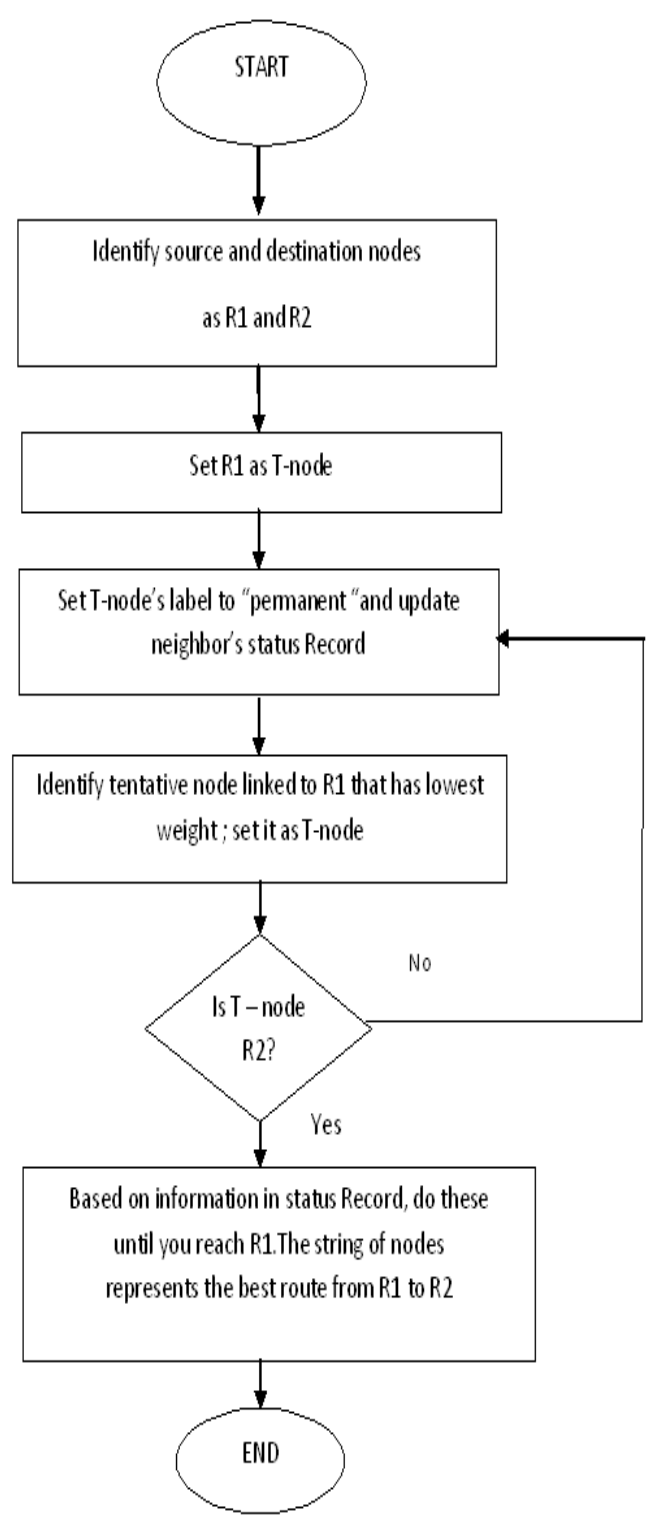

Fig 2: Flow Chart to find shortest Path using I R Algorithm

\section{COMPARISON OF IRA WITH CONVENTIONAL METHODS}

In this paper the IRA method is compared with ACO (Ant Colony Optimization) and PSO (Particle Swarm Optimization) sensor positioning algorithms. In this paper the Iterative Routing Algorithm is used to estimate the sensor positioning, computational cost, number of iterations, radio range, number of nodes, ranging error and also to find the shortest path between the source and destination nodes. In ACO algorithm, the estimation of senor positioning and finding the shortest path from one node to another node has been proposed [8]. In this algorithm number of iterations are more and computational cost is high compared to our approach. Through PSO algorithm a distributed iterative localization is explained in WSNs [10]. In this algorithm the deployment of sensor nodes is less and computational cost is high. In our approach as the radio range increases the number of nodes placed in the coverage area also increases.

\subsection{ACO Algorithm}

In ACO algorithm Gupta et al. proposed Sensor Positioning and Routing problems to find the shortest path between source node and destination node [8]. In Senor Positioning and routing problem, a set of artificial ants (nodes) are simulated from source to destination. Firstly, taking the information from the routing table the forward ants select nodes randomly and the ants which are successful in reaching the destination update the pheromone deposit at the edges visited by them as shown in Fig.3 and shortest path is as shown in Fig.4.

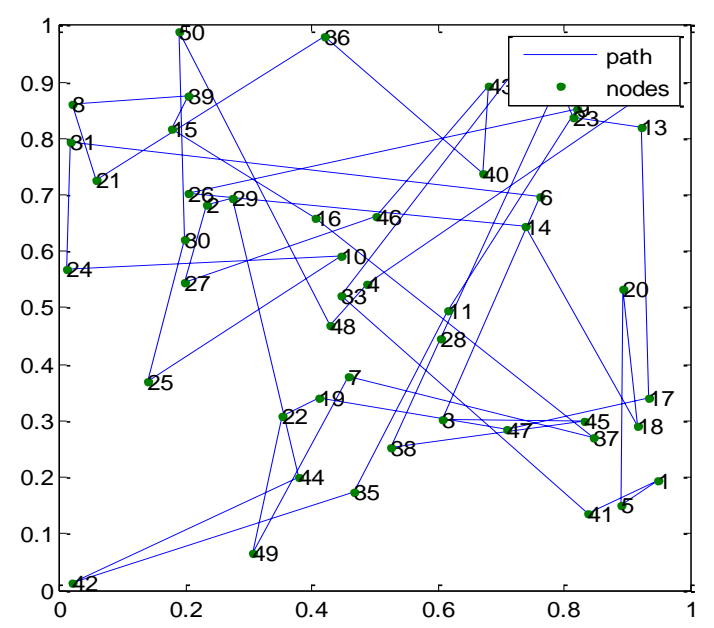

Fig 3: Positions estimated by ACO

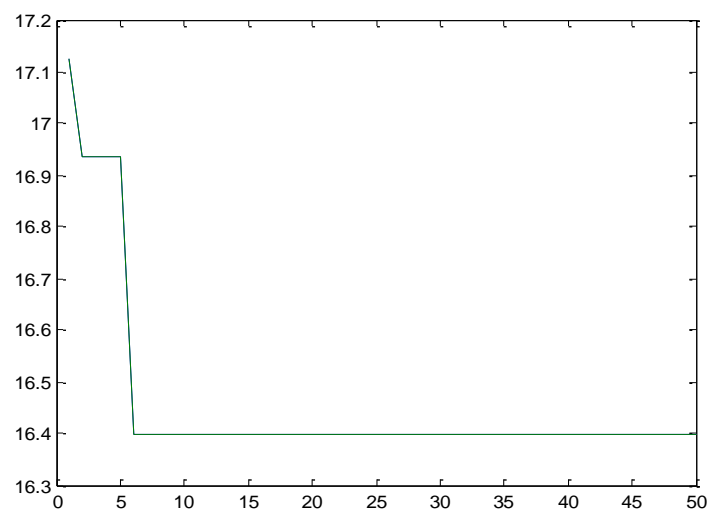

Fig 4: Shortest path using ACO

\subsection{PSO Algorithm}

In the PSO conventional method by Kulkarni et al. a distributed iterative localization algorithm PSO-iterative in WSNs [9-10] is proposed. Each target node that has three or more beacons in its hearing range runs PSO to minimize the localization error. Nodes that get localized act as beacons for 
other nodes. This continues iteratively routing algorithm of all the nodes are localized. This method does not require the transmission of range measurement by each node to a central node. Besides, it can localize all nodes that have three localized nodes or beacons in their range. As the localization iterations pass by, a node may get more number of references for localization, which is a serious problem in WSNs [11], the situation that references are near-collinear as shown in Fig.5. However, the proposed method in this paper is prone to error accumulation away; to find the shortest path, to estimate node positioning, radio range and computational cost.

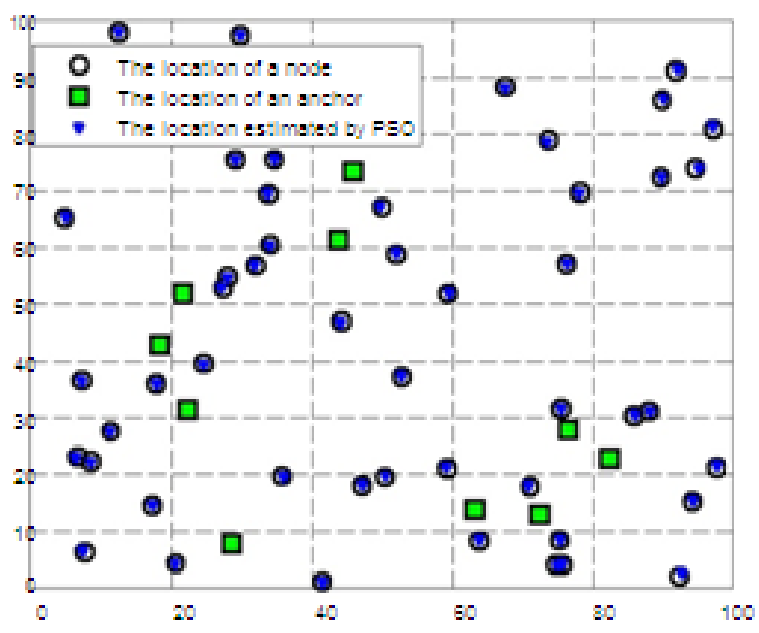

Fig 5: Positions estimated by PSO

\subsection{R Algorithm}

The IRA Sensor Positioning and Routing method is used to estimate all sensors and to find relative locations of sensors by applying IRA with low cost and high error tolerance. The routing algorithm is stored in the routers memory. The routing algorithm is a major factor in the performance of position estimation and distance measurement in the sensor field. The purpose of the routing algorithm is to make decisions for the router concerning the best paths for estimating the positions of anchors. The router uses the routing algorithm to compute the path that would best serve to find the shortest path between the source and destination nodes as shown in Fig. 6 and Fig.7. In this approach the computational cost is less when compared to the existing methods like ACO and PSO.

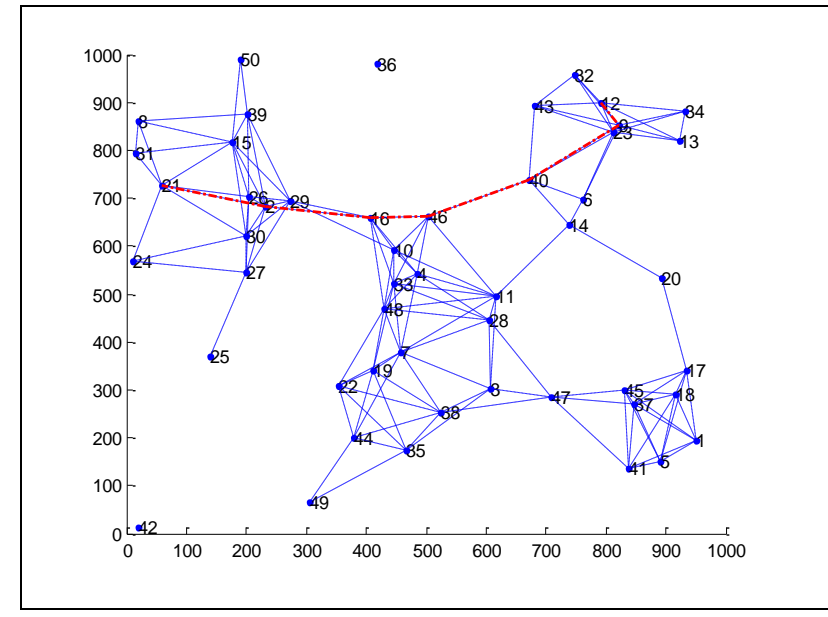

Fig 6: Positions estimated and Shortest path using IRA with $4 \%$ error

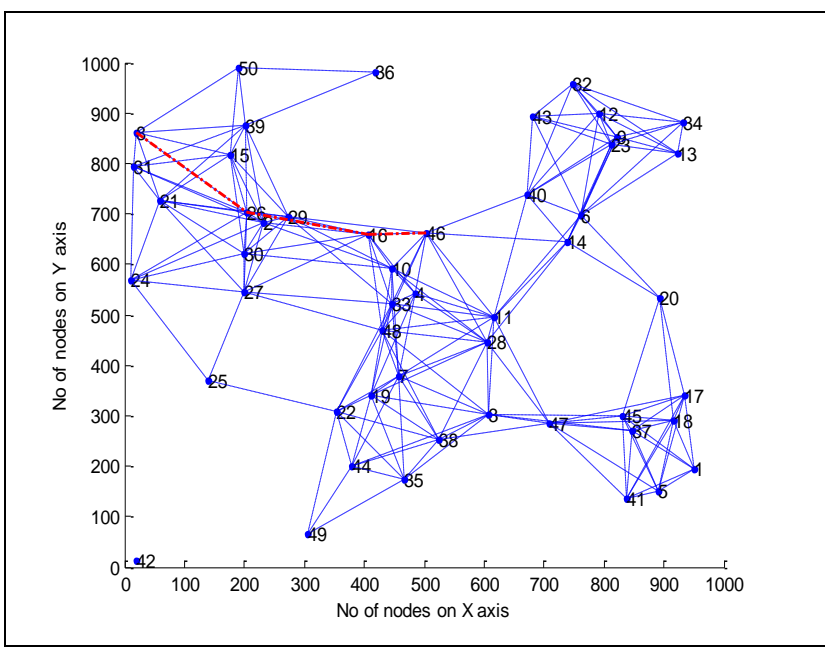

Fig 7: Positions estimated and Shortest path using IRA with $2 \%$ error

\section{SIMULATION RESULTS}

In the MATLAB simulation, a random network of 50 nodes was created for a radio range 200 and 250 and an IRA is used to find the blue dots represent the true position of unknowns; anchors are indicted explicitly. A line between two nodes indicates a radio link. In the figure the Red dotted line represent the shortest path between the anchors that means from source node to destination node. The source and destination nodes are shown in above Figures 5 and 6 . The computational cost for this method is 1.35 and 0.75 . In this method the main advantage is computational cost is reduced for every iteration. 


\section{CONCLUSIONS AND FUTURE WORK}

This paper compares IRA with existing methods like ACO and PSO algorithms for determining coordinates of the nodes in a WSN in a distributed and iterative fashion. The localization problem is treated as a multidimensional optimization problem. IRA sensor positioning algorithm proposed here has the advantage of reduced computational cost, high radio range which increases coverage area in WSNs applications. The paper briefly outlines the algorithms and presents a comparative analysis of their results as shown in the Table.1. The results show that the proposed algorithms have a trade off issue. This work can be extended in several directions. It shows that the proposed algorithm works well for near uniform radio propagation. However, in the real world, radio propagation in indoors and in cluttered circumstances is far from uniform. Local distance estimation can also be found out using this algorithm. Further simulations will be needed to determine reduction of range errors using MDS algorithms. An IRA builds the local positions and routings of the estimated sensors for applications which require absolute coordinates of nodes. It is used to high radio range and also extended to 3-D approach in WSN.

Table 1. Comparative analysis of Iterative Routing Algorithm with Conventional Methods

\begin{tabular}{|c|l|l|l|l|c|c|c|}
\hline $\begin{array}{l}\text { Title of } \\
\text { Positioning } \\
\text { Algorithm }\end{array}$ & $\begin{array}{l}\text { Description of } \\
\text { Algorithm }\end{array}$ & $\begin{array}{l}\text { Radio } \\
\text { Range }\end{array}$ & $\begin{array}{l}\text { Ranging } \\
\text { Error }\end{array}$ & $\begin{array}{l}\text { Computation } \\
\text { al Cost }\end{array}$ & $\begin{array}{l}\text { No of } \\
\text { Iterations }\end{array}$ & $\begin{array}{l}\text { Shortest } \\
\text { Path }\end{array}$ & $\begin{array}{l}\text { Number of } \\
\text { nodes }\end{array}$ \\
\hline $\begin{array}{c}\text { Ant Colony } \\
\text { [6] }\end{array}$ & $\begin{array}{l}\text { Optimize routing } \\
\text { paths, Effective } \\
\text { Multi-path data } \\
\text { transmission }\end{array}$ & 100 & 15 & 9.5 & 150 & 16 & 50 \\
\hline $\begin{array}{c}\text { Particle Swarm } \\
\text { Optimization } \\
{[2]}\end{array}$ & $\begin{array}{l}\text { Node deployment, } \\
\text { localization, } \\
\text { Energy-aware } \\
\text { clustering and data- } \\
\text { aggregation }\end{array}$ & 100 & 5 & 10.7 & 50 & - & 30 \\
\hline $\begin{array}{c}\text { Iterative } \\
\text { Routing }\end{array}$ & $\begin{array}{l}\text { Node deployment, } \\
\text { Estimate position of } \\
\text { the nodes, shortest } \\
\text { path, computational } \\
\text { cost, radio range and } \\
\text { [Present Work] }\end{array}$ & 200 & 4 & 1.35 & 50 & 6 & $>50$ \\
\hline
\end{tabular}

\section{ACKNOWLEDGMENTS}

The author gratefully acknowledges to Annamacharya Institute of Technology and Sciences for the Environment providing facilities to this work. Finally, a thank you goes to the reviewers for their insightful suggestions to improve the quality of this paper.

\section{REFERENCES}

[1] N.Pushpalatha and Dr.B.Anuradha, "A TwoDimensional IR Algorithm for Position Estimation in Wireless Sensor Networks", International Journal of Computer Science And Technology, ISSN: 0976-8491(Online) | ISSN: 2229-4333 (Print) IJCST Vol. 3, Issue 2, April June 2012. Pp:35-39.

[2] N.Pushpalatha and Dr.B.Anuradha, "Shortest path position estimation between source and destination nodes in wireless sensor networks with low cost", International Journal of Emerging Technology and Advanced Engineering (ISSN 2250-2459, Volume 2, Issue
4, April 2012). Pp:6-12

[3] N. Pushpalatha and Dr.B.Anuradha, "Distribution of Nodes on Square Method for Wireless Sensor Networks", in International Journal of Computer Science and Telecommunications [Volume 3, Issue 1, January 2012].

[4] N.Pushpalatha and Dr.B.Anuradha, "Study of Various Methods of Wireless Ad-hoc Sensor Networks using Multidimensional Scaling for Position Estimation" Global Journal Engineering and AppliedSciences-ISSN22492631(online): 2249-2623(Print) GJEAS Vol.1(3) , 2011. Pp:76-81

[5] Mohammad, I.; Imad, M. Handbook of Sensor Networks; CRC Press: London, 2005; pp. 117140. 
[6] Heinzelman,W.; Chandrakasan, A Balakrishnan, H. Energy-efficient Communication Protocol for Wireless Microsensor Networks. In Proceedings of the 33rd Annual Hawai International Conference on System Sciences, 4-7 January 2000; pp. 1-10.

[7] Al-Karaki, J.N.; Kamal, A.E. Routing Techniques in Wireless Sensor Networks: a Survey. IEEE Wireless Commun. 2004, 11, 628 .

[8] Anuj K. Gupta, Harsh Sadawarti, and Anil K. Verma, "MANET Routing Protocols Based on Ant Colony Optimization", International Journal of Modeling and Optimization, Vol. 2, No. 1, February 2012.

[9] Raghavendra V. Kulkarni, Senior Member, IEEE, and Ganesh Kumar Venayagamoorthy, Senior Member, IEEE, "Particle Swarm Optimization in Wireless Sensor Networks: A Brief Survey"

[10] Raghavendra V. Kulkarni, Ganesh K. Venayagamoorthy Maggie X. Cheng, "BioInspired Node Localization in Wireless Sensor Networks",978-1-4244-2794-9/09/\$25.00 (C)2009 IEEE

[11] Wu-chun Feng. Securing wireless communication in heterogeneous environments [C], MILCOM, 2002, 2: 1101-1106.

\section{AUTHORS PROFILE}

N.Pushpalatha completed her B.Tech at JNTU, Hyderabad in 2004 and M.Tech at A.I.T.S., Rajampet in 2007. Presently she is working as Assistant Professor of ECE, Annamacharya Institute of Technology and Sciences Tirupati since 2006. She has guided many B.Tech projects. Her Research area includes Data Communications and Ad-hoc Wireless Sensor Networks.

Dr.B.Anuradha is working as Associate Professor in the Department of ECE, at Sri Venkateswara University College of Engineering since 1992. She has guided many B.Tech and M.Tech projects. At present Five Scholars are working for PhD. She has published a good number of papers in journals and conferences. 